\title{
In-situ TEM Study of Thermal Stabilities of Metastable Silicon Phases
}

\author{
Daibin Ge, Vladislav Domnich, Yury Gogotsi
}

\section{A.J. Drexel Nanotechnology Institute and Department of Materials Engineering, Drexel University, 3141 Chestnut Street, Philadelphia, PA 19104}

The cubic diamond structure of silicon ( $\mathrm{Si}-\mathrm{I})$ undergoes a phase transformation to a tetragonal structure (Si-II) at a pressure level of 11.2 12 GPa. This transition is not reversible; a mixture of body-centered cubic Si-III $(b c 8)$ and rhombohedral $\mathrm{Si}$-XII $(r 8)$ structures or amorphous silicon $(a-\mathrm{Si})$ may form during pressure release [1]. In the last decade, depth-sensing indentation has proven to be a powerful tool for studying phase transformations in silicon under highly deviatoric stresses. However, the transmission electron microscopy (TEM) analysis of various Si structures formed during nanoindentation has been largely inhibited by the difficulties involved in sample preparation. Recently, with the aid of focused ion beam and other advanced TEM sample preparation techniques, a few groups have performed successful TEM studies on residual silicon indentations [2-6]. However, the structures formed under load and the exact transformation mechanisms between different phases are still not fully understood. Furthermore, the thermal stabilities of metastable phases (such as Si-III and Si-XII) formed within nanoindentations have not been reported. In this paper, a plan-view TEM work on silicon nanoidentations is presented. This work is then used to discuss phase transformation mechanisms during unloading and to investigate the stability of metastable silicon phases during heating in TEM.

The sample preparation of the plan-view samples started with two nanoindentation lines crossed on a polished silicon wafer with thickness of $50 \mu \mathrm{m}$. Nanoindentaitons were made using a Nano Indenter XP ${ }^{\circledR}$ (MTS Corporation) and a Berkovich indenter with a nominal tip radius of $70 \mathrm{~nm}$ under identical conditions: maximum load of $50 \mathrm{mN}, 60 \mathrm{~s}$ loading and 3 min unloading time. The slow unloading rate was chosen to favor the formation of the crystalline metastable phases, $\mathrm{Si}$-XII and $\mathrm{Si}$ III [1]. To protect the indentation surface from redeposition during single-side ion milling, a layer of nail polish was deposited onto the indented surface. Interrupted ion milling was adopted to minimize the possible structural modification in a PIPS station. After ion milling, the sample was examined using a Philips 420 and a JEOL 2010F TEM/STEM with a point-to-point resolution of $0.23 \mathrm{~nm}$.

An analysis of selected area diffraction (SAD) patterns suggested the presence of a mixture of metastable phases $\mathrm{Si}$-III and $\mathrm{Si}$-XII within the residual indents. However, due to structural similarities between Si-III and Si-XII ( $r 8$ is a rhombohedral distortion of $b c 8$ ), the two phases yield very similar electron diffraction patterns. The spot splitting in both tangential and radial directions in some SAD patterns indicated the presence of a pseudo-cubic structure (Si-XII) in addition to the perfect cubic phase $\mathrm{Si}$-III in the residual indentation. Uneven interfaces between the crystalline metastable phases and the amorphous zone surrounding them suggested a lattice-distortion-induced amorphization mechanism. Lattice rotations by introducing several dislocations were observed in our high resolution TEM images.

The results of our in-situ investigation on the thermal stability of the metastable phases produced by nanoindentation are summarized in Fig. 1. The Si-XII and Si-III phases were found to be unstable under a high energy electron dose during our high resolution TEM imaging, although the Si-III phase has been reported to last for years at room temperature [7]. Both in situ high resolution observation and SAD patterns showed that the perfectly ordered metastable phases Si-III/Si-XII had 
collapsed into an amorphous structure after annealing at $200^{\circ} \mathrm{C}$ for 5 minutes. It is likely that the crystalline lattice had been locally distorted and rotated and that due to the heterogeneity of the residual strain and shear stress within the residual nanoindentation, it finally decomposed into an amorphous structure during annealing. Such a hypothesis is supported by the SAD comparison between the single diffraction spots pattern before heating and the strongly diffused pattern with long streaks after in situ annealing (Fig. 1(c)).

References:

○[1] V. Domnich and Y. Gogotsi, Rev. Adv. Mater. Sci. 3 (2002) 1.

○[2] I.Zarudi et al., App. Phys. Lett. 82 (2003) 874.

○[3] J.E. Bradby et al., App. Phys. Lett. 77 (2000) 3749.

o[4] A.B. Mann et al., J. Mater. Res., 15 (2000) 1754.

o [5] S.J. Lloyd et al., J. Mater. Res., 16 (2001) 3347.

○[6] D. Ge et al, J. App. Phys., 93 (2003) in press.

$\circ$ [7] G. Weill et al, Semicond. Sci. Tech. 4, 280-282 (1989).

○[8] The authors appreciate the financial support from NSF under Grant No. DMR-0196424. Electron Microscopes at the Regional Materials Characterization Facility of the University of Pennsylvania were used.

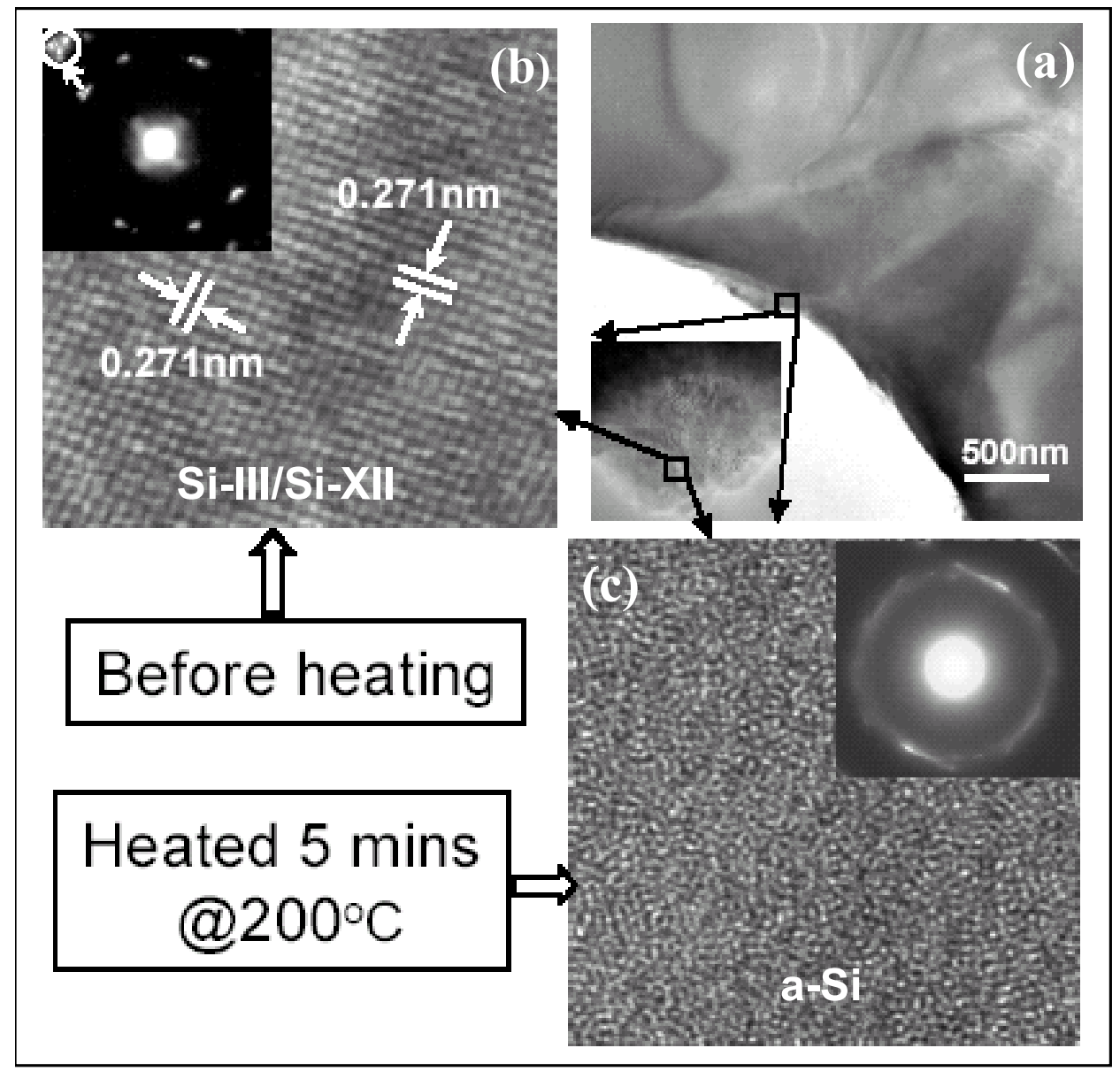

Fig. 1(a) BF image of a nanoindentation; (b) high resolution image of an edge area before/ (c) after in situ annealing at $200^{\circ} \mathrm{C}$ for 5 mins, and the corresponding SAD patterns. 